\title{
A amamentação e a alimentação complementar de filhos de mães adolescentes são diferentes das de filhos de mães adultas?
}

\author{
Are breastfeeding and complementary feeding of children of adolescent \\ mothers different from those of adult mothers?
}

\author{
Maria L.F. Vieira ${ }^{1}$, João L.C. Pinto e Silva ${ }^{2}$, Antônio A. Barros Filho ${ }^{3}$
}

\section{Resumo}

Objetivos: estudar a amamentação ao longo do primeiro ano de vida, e o tipo de alimentos complementares utilizados no final do primeiro ano de vida em filhos de mães adolescentes, e comparar com os filhos de mães adultas.

Métodos: estudo tipo coorte, ambidirecional, no qual as crianças foram selecionadas nos arquivos do CAISM/UNICAMP e avaliadas com um ano de idade. Estudados 122 filhos de adolescentes e 123 filhos de adultas, nascidos a termo, pesando $\geq 2.500 \mathrm{~g}$. Entrevistas realizadas nas casas ou no CIPED/UNICAMP, quando as crianças tinham um ano. Foram usados teste do qui-quadrado e de Fisher, $\alpha=5 \%$, análise de sobrevida da amamentação pelo método de Kaplan-Meier e teste de Wilcoxon (Breslow) para comparar as curvas de amamentação exclusiva, predominante, completa e total.

Resultados: $94,3 \%$ dos filhos de adolescentes e 95,9\% dos filhos de adultas saíram da maternidade amamentados ( $\mathrm{p}=0,544)$. A mediana de amamentação exclusiva foi de 90 dias para ambos os grupos. Com um ano de idade, $35,3 \%$ e $28,5 \%$ dos filhos de mães adolescentes e adultas, respectivamente, continuavam amamentados $(\mathrm{p}=0,254)$ : leite materno $11,5 \%$ x $8,9 \%$ e aleitamento misto $23,8 \% \times 19,5 \%(\mathrm{p}=0,519)$. Os filhos de adolescentes ingeriram menos carne diariamente, comparados aos filhos de adultas $(13,9 \%$ x 26,0\%; Fisher: p=0,031). Ovo não é consumido por $11,5 \%$ x $19,5 \%$ dos filhos de adolescentes e de adultas, mas sugere ser mais consumido pelos filhos de adolescentes $(\mathrm{p}=0,082)$.

Conclusões: o tempo de amamentação e o seu padrão foram semelhantes entre os filhos de mães adolescentes e de mães adultas. A alimentação complementar também foi similar, com exceção de um menor consumo de carnes e um maior consumo de ovos entre os filhos de mães adolescentes.

J Pediatr (Rio J) 2003;79(4):317-24: amamentação, alimentos complementares, curvas de amamentação.

\begin{abstract}
Objectives: to study breastfeeding during the first year of life and the kind of complementary food provided at one year of life to children of adolescent mothers. To compare these data with breastfeeding and complementary food received by children of adult mothers.

Method: a dual cohort was performed. Children were selected from the files of CAISM/UNICAMP and assessed when they were one year old. This study consisted of 122 children born from adolescent mothers and 123 children born from adult mothers - full-term births, birthweight was $2,500 \mathrm{~g}$ or higher. When the children were one year old, the mothers were interviewed at home or at CIPED/UNICAMP. The results were compared using the chi-square test and the Fisher's test; $\alpha=5 \%$; the Kaplan-Meier method was used to analyze the duration of breastfeeding and the Wilcoxon test (Breslow) to compare the exclusive, predominant, full and total breastfeeding curves.

Results: $94.3 \%$ of children of adolescent mothers and $95.9 \%$ of children of adult mothers left the maternity hospital being breastfed $(\mathrm{p}=0,544)$. The median exclusive breastfeeding duration for both groups was 90 days. After completing one year, $35.3 \%$ and $28.5 \%$ of children of adolescent and adult mothers, respectively, continued breastfeeding $(\mathrm{p}=0.254)$ : only breastfeeding $11.5 \%$ x $8.9 \%$ and mixed feeding $23.8 \% \times 19.5 \%(\mathrm{p}=0.519)$. Meat intake by children of adolescent mothers was lower than that of children of adult mothers (13.9\% x 26.0\%; Fisher's test: $\mathrm{p}=0.031$ ). With regard to egg intake, $11.5 \% \times 19.5 \%$ of children of adolescent mothers and adult mothers did not eat egg but the results suggested that the egg intake of children of adolescent mothers was higher $(\mathrm{p}=0.082)$.

Conclusions: duration and pattern of breastfeeding were similar between children of adolescent mothers and of adult mothers. The complementary nutrition was similar, except for a lower intake of meat and a higher intake of eggs among the children of adolescent mothers.
\end{abstract}

J Pediatr (Rio J) 2003;79(4):317-24: breastfeeding, complementary nutrition, breastfeeding curves.

1. Doutoranda do Curso de Pós-Graduação em Saúde da Criança e do Adolescente da UNICAMP, Profa do Dep. de Toco-Ginecologia e Pediatria da UFAL.

2. Prof. Titular de Obstetrícia do Departamento de Toco-Ginecologia da Faculdade de Ciências Médicas da UNICAMP.

3. Prof. Doutor do Departamento de Pediatria da Faculdade de Ciências Médicas da UNICAMP. 
A adolescência é um período crítico do ser humano, no qual acontecem intensas transformações biológicas e psicossociais ${ }^{1}$. A ocorrência de gravidez e maternidade nessa fase da vida implica em somatória de mudanças que exigem um repensar no futuro com um filho, geralmente, não desejado. Gravidez e maternidade na adolescência são consideradas um grave problema de saúde pública em todo o mundo, principalmente em famílias de baixa renda, população de risco para agravos à saúde ${ }^{2}$.

Em países em desenvolvimento, inclusive o Brasil, trabalhos têm mostrado preocupação com a prevenção da desnutrição infantil por meio da promoção da saúde, incentivando à amamentação, principalmente em mães adolescentes. A OMS, o UNICEF e vários estudos destacam a necessidade e a importância de promover o aleitamento materno exclusivo, como forma eficaz de prevenir doenças infecciosas (gastrointestinais, respiratórias e otites médias), enterocolite necrotisante, retocolite ulcerativa, Doença de Crohn; proteger contra alergias tardias, diabetes mellitus insulino-dependentes, sobrepeso e disfunções cognitivas; e favorecer o crescimento e o desenvolvimento infanti ${ }^{3-7}$. Já está bem sedimentado o conhecimento das vantagens da amamentação e do leite humano para a saúde, crescimento e desenvolvimento da criança ${ }^{5}$, porém Gouvêa ${ }^{4}$ reforça também a importante contribuição da amamentação no desenvolvimento da fala. Nos serviços de saúde melhor organizados do Brasil, equipes multiprofissionais trabalham a auto-estima de gestantes/puérperas adolescentes, incentivando-as a assumirem responsabilidades pessoais $\mathrm{e}$ com seus bebês, fortalecendo o vínculo mãe-filho e favorecendo a amamentação ${ }^{4}$.

A Declaração Innocenti ${ }^{8}$, em 1990, preconizou a amamentação exclusiva nos primeiros quatro a seis meses de vida, como objetivo ideal de saúde e nutrição infantil. Atualmente, a OMS recomenda: (1) amamentação exclusiva durante os seis primeiros meses de vida; (2) alimentação complementar oportuna e adequada; e (3) manutenção do aleitamento materno complementado até os dois anos de idade ou mais ${ }^{9,10}$. A última Pesquisa Nacional sobre Demografia e Saúde ${ }^{5}$, realizada em 1996, mostra que o aleitamento materno entre nós está aquém das recomendações da OMS, com mediana de amamentação exclusiva de apenas um mês, duração total de amamentação de sete meses, e o aleitamento continuado um ano em $41,0 \%$ das crianças 9 .

Estudo de Almroth ${ }^{7}$ et al. mostra que a amamentação exclusiva não é uma prática em Lesoto, onde mães, avós e até enfermeiras dos serviços de saúde acreditam que oferecer água às crianças é importante e necessário, promovendo a amamentação predominante. Os hábitos culturais favorecem essa prática, daí a ampla variabilidade na prevalência da amamentação predominante no Brasi1 ${ }^{11-13}$.

Desde março de 2001, após extensa revisão da literatura, a OMS adotou a idade de seis meses para a introdução dos alimentos complementares ${ }^{14}$. Em Honduras, estudos de intervenção aleatorizados não mostraram vantagens no crescimento de crianças que iniciaram alimentos comple- mentares aos quatro meses de idade, comparadas com crianças amamentadas exclusivamente ao seio até o $6^{\circ}$ mês de vida ${ }^{15}$. Mehta ${ }^{16}$ et al. afirmaram que lactentes consumindo alimentos industrializados têm menor ingestão de proteínas e gorduras, mas sem efeito no crescimento, e que a introdução precoce de sólidos na dieta não altera o crescimento e a composição corporal durante o primeiro ano de vida. Burrows ${ }^{17}$ et al. não encontraram diferença significativa entre filhos de mães adolescentes e de adultas na ingestão de proteínas, carboidratos e lipídeos, enquanto para Carruth ${ }^{18}$ et al., os filhos de mães adolescentes ingerem mais gorduras e iniciam a se alimentar com a própria mão e com cereais mais precocemente que os filhos de adultas, sem diferença para frutas, vegetais e carne.

Este estudo objetivou estudar a amamentação durante o primeiro ano de vida e o tipo de alimentos complementares utilizados no final do primeiro ano de vida em filhos de mães adolescentes; comparar as curvas de amamentação exclusiva, predominante, completa e total; identificar a taxa de aleitamento continuado um ano; e comparar consumo de carnes, vísceras, ovos, legumes, frutas, cereais e tipo de alimentação láctea entre os filhos de mães adolescentes e de adultas, com um ano de idade.

\section{Material e métodos}

Estudo tipo coorte, ambidirecional, no qual as crianças foram selecionadas, retrospectivamente, e analisadas, prospectivamente, com um ano de idade, após aprovação do protocolo de pesquisa pelo Comitê de Ética em Pesquisa do Centro de Atenção Integral à Saúde da Mulher - CAISM/ UNICAMP. Esta é parte de um estudo maior sobre crescimento e desenvolvimento de filhos de mães adolescentes, no qual o cálculo do tamanho amostral foi baseado nas variáveis quantitativas de interesse (peso, comprimento, perímetros cefálico e braquial e pregas cutâneas tricipital e subescapular), usando-se referência de Frisancho ${ }^{19}$, por meio da fórmula:

$$
n=\frac{2(\sigma)^{2}}{(d)^{2}} \cdot f(\alpha, b)
$$

onde $\boldsymbol{\sigma}$ é o desvio padrão das medidas antropométricas usadas, $\mathbf{d}$ é a diferença considerada significativa entre os grupos, e $\boldsymbol{f}$ é a função do erro tipo I e tipo II. Após os cálculos, para as análises propostas, seriam necessários 102 sujeitos em cada grupo, porém conseguiu-se localizar e avaliar 122 filhos de mães adolescentes e 123 filhos de mães adultas. A amostra foi seqüencial, ou seja, as crianças foram selecionadas nos arquivos do CAISM/UNICAMP por ordem de nascimento, conforme os critérios: nascidos a termo, partos únicos, pesando $\geq 2.500$ gramas, sem malformações congênitas, síndromes ou doenças interferentes no crescimento e desenvolvimento; mães menores de 20 anos de idade por ocasião do parto e mães adultas entre 20 e 30 anos completos; todas primíparas e sem doença clínica ou mental grave, que pudesse interferir nos cuidados com o 
filho. As crianças selecionadas eram buscadas, e as localizadas foram avaliadas em suas residências ou no CIPED (Centro de Investigação em Pediatria) da UNICAMP até completar o tamanho de amostra calculado, conseguindose $20 \%$ a mais em cada grupo estudado. Feito busca ativa para localizar as mães e seus filhos em bairros periféricos da grande Campinas-SP e nove cidades adjacentes. Como as perdas foram decorrentes de não localização do endereço registrado no CAISM ou de mudança de endereço e/ou cidade e/ou Estado, e sendo semelhantes em ambos os grupos, (quatro de mães adultas e três de mães adolescentes) não consideramos que houve viés de seleção, e essas crianças foram substituídas por outras selecionadas subseqüentemente, conforme os critérios de inclusão. Devido às dificuldades de acesso e localização dos endereços das mães do estudo, a coleta de dados estendeu-se de outubro de 1999 a abril de 2001, realizada por apenas um entrevistador/pesquisador.

Todas as mães assinaram um termo de consentimento informado, conforme recomendação da Resolução no 196 de 10/10/1996 sobre pesquisa envolvendo seres humanos, do Conselho Nacional de Saúde do Ministério da Saúde, e escolheram horário e local para a coleta dos dados e a entrevista individual.

Foram usadas: (1) ficha do CAISM/UNICAMP - dados maternos gineco-obstétricos e neonatais; (2) ficha da criança - informações do desenvolvimento neuropsicomotor, além das medidas antropométricas + imunizações desde o nascimento até um ano de idade, transcritas da "Caderneta da Criança"; e (3) ficha da visita domiciliar - dados socioeconômicos, morbidade e inquérito alimentar.

A escolaridade foi definida como adequada à idade levando-se em consideração que, aos sete anos, toda criança deve estar cursando a primeira série do Ensino Fundamental, cuja $8^{a}$ série deve estar sendo cursada aos 14/15 anos de idade, segundo o Ministério da Educação; e as adultas, por terem mais de 20 anos, devem ter o Ensino Médio completo. A situação conjugal definida como "unida" para as casadas ou amasiadas e "não unida" quando solteiras, separadas/desquitadas ou viúvas. O trabalho materno foi categorizado em do lar ("dona de casa") e fora do lar (todos os trabalhos remunerados). A renda mensal familiar foi dividida pelo número de pessoas da casa e classificada em < 0,5; 0,5 a 1,0 (exclusive); 1,0 a 2,0 (exclusive); e $\geq 2,0$ salários-mínimos per capita.

Amamentação exclusiva significa que a criança ingere somente leite materno, sem suplementos; enquanto amamentação predominante é a ingestão de leite materno + água e/ou chás e/ou sucos e/ou sais de reidratação oral. Amamentação completa (full breastfeeding) = amamentação exclusiva + amamentação predominante; e amamentação total $=$ amamentação exclusiva e/ou predominante + amamentação complementada com outros alimentos. Aleitamento misto = mamar leite materno e leite de vaca. Suplementos alimentares foram considerados: água, chás e/ou substitutos do leite materno, consumidos nos primeiros meses de vida; enquanto complementos alimentares são alimentos para complementar o leite materno a partir do sexto mês de vida $^{9,13}$.

Na parte alimentar deste estudo, investigou-se amamentação durante o primeiro ano de vida e alimentos complementares consumidos ao final do primeiro ano de idade. Perguntado às mães: (1) se amamentaram, considerando sim quando a criança saiu da maternidade mamando no peito e continuou amamentada por mais de 20 dias; (2) quanto tempo (em dias) foi a amamentação exclusiva, completa e total; (3) com quantos meses de idade introduziu-se água e/ou chás; (4) se seus filhos ainda mamavam no peito, na época da entrevista; (5) que tipo de aleitamento seus filhos tinham com um ano de idade: materno, misto ou leite de vaca; e (6) se seus filhos, com um ano de idade, nunca ingeriram, ingeriam diariamente ou ocasionalmente carnes (bovina, peixe e frango), vísceras (fígado de boi ou frango), ovos, cereais, legumes/verduras e frutas. Diariamente, quando a criança ingeria todos os dias os complementos alimentares; e ocasionalmente, quando a freqüência de ingestão dos alimentos investigados era irregular.

Foram usados o teste de McNemar para comparações tipo antes e depois (situação conjugal das mães adolescentes e adultas ao engravidar e quando o filho estava com um ano de idade); o teste do qui-quadrado ou exato de Fisher, para comparar proporções; e o teste de Wilcoxon (Breslow), para comparar curvas de sobrevida da amamentação. A análise do tempo de sobrevida da amamentação exclusiva, realizada pelo método de Kaplan-Meier, considerando como evento a interrupção da amamentação com leite materno apenas. Aceito $\alpha$ de $5 \%$.

\section{Resultados}

A Tabela 1 mostra algumas características das mães adolescentes e adultas, destacando a situação conjugal, o tipo de trabalho materno e a renda per capita que se apresentaram diferentes entre os grupos. As mães adolescentes, com mais freqüência, tinham renda per capita menor que um salário-mínimo, quando comparadas às mães adultas. As adolescentes trabalhavam menos "fora do lar" que as adultas; ao engravidar, a situação conjugal "não unida" foi maior no grupo de adolescentes, e durante o primeiro ano de vida de seus filhos, $25,4 \%$ dessas mães casaram-se ou se amasiaram, mudando significativamente para a situação "unida".

A Tabela 2 descreve as características das crianças da amostra, cujo comprimento ao nascer, peso e IMC com um ano de idade foram menores nos filhos de mães adolescentes, quando comparados aos filhos de adultas, usando-se ANCOVA, corrigindo o efeito do sexo.

A Tabela 3 mostra que não há diferença em ser filho de mãe adolescente ou adulta com relação à amamentação, aleitamento continuado um ano, tipos de alimentação láctea 
Tabela 1 - Características das mães adolescentes e adultas

\begin{tabular}{|c|c|c|c|}
\hline Características maternas & $\begin{array}{l}\text { Adolescentes } \\
(\mathbf{n}=122)\end{array}$ & $\begin{array}{l}\text { Adultas } \\
(\mathbf{n}=123)\end{array}$ & $\mathbf{p}$ \\
\hline Média de idade (anos) & $17,0 \pm 1,6$ & $23,3 \pm 2,8$ & \\
\hline $\begin{array}{l}\text { Escolaridade } \\
\% \text { adequada à idade }\end{array}$ & $26,2 \%(32)$ & $35,8 \%(44)$ & $0,106^{*}$ \\
\hline $\begin{array}{l}\text { Situação conjugal ao engravidar } \\
\text { Com companheiro } \\
\text { Sem companheiro }\end{array}$ & $\begin{array}{l}47,5 \%(58) \\
52,5 \%(64)\end{array}$ & $\begin{array}{l}75,6 \%(93) \\
24,4 \%(30)\end{array}$ & $0,001 *$ \\
\hline $\begin{array}{l}\text { Situação conjugal quando filho com um ano } \\
\text { Com companheiro } \\
\text { Sem companheiro }\end{array}$ & $\begin{array}{l}72,9 \%(89) \\
27,1 \%(33)\end{array}$ & $\begin{array}{l}86,2 \%(106) \\
13,8 \%(17)\end{array}$ & 0,010 \\
\hline $\begin{array}{l}\text { Tipo de trabalho materno } \\
\text { Do lar } \\
\text { Fora de casa }\end{array}$ & $\begin{array}{l}77,9 \%(95) \\
22,1 \%(27)\end{array}$ & $\begin{array}{l}64,2 \%(79) \\
35,8 \%(44)\end{array}$ & 0,019 * \\
\hline $\begin{array}{l}\text { Tabagismo } \\
\quad<10 \text { cigarros/dia } \\
\geq 10 \text { cigarros/dia } \\
\text { Não fumou na gestação }\end{array}$ & $\begin{array}{l}8,2 \%(10) \\
4,1 \%(5) \\
87,7 \%(107)\end{array}$ & $\begin{array}{l}8,1 \%(10) \\
3,3 \%(4) \\
88,6 \%(109)\end{array}$ & $0,954^{\dagger}$ \\
\hline $\begin{array}{l}\text { Pré-natal } \\
\quad<6 \text { consultas } \\
\geq 6 \text { consultas } \\
\text { Não fez } \\
\text { Não lembra }\end{array}$ & $\begin{array}{l}18,0 \%(22) \\
76,2 \%(93) \\
2,5 \%(3) \\
3,3 \%(4)\end{array}$ & $\begin{array}{l}8,1 \%(10) \\
88,7 \%(109) \\
0,8 \%(1) \\
2,4 \%(3)\end{array}$ & $0,024^{\dagger}$ \\
\hline $\begin{array}{l}\text { Início de pré-natal (meses) } \\
\quad<4^{\circ} \text { mês } \\
\geq 4^{\circ} \text { mês } \\
\text { Não lembra } \\
\text { Não fez PN }\end{array}$ & $\begin{array}{l}47,5 \%(58) \\
41,0 \%(50) \\
9,0 \%(11) \\
2,5 \%(3)\end{array}$ & $\begin{array}{l}60,2 \%(74) \\
33,3 \%(41) \\
5,7 \%(7) \\
0,8 \%(1)\end{array}$ & $0,106^{\dagger}$ \\
\hline $\begin{array}{l}\text { Salários-mínimos per capita } \\
\quad<0,5 \\
\quad 0,5 \mid-1,0 \\
\quad 1,0 \mid-2,0 \\
\quad \geq 2,0\end{array}$ & $\begin{array}{l}10,0 \%(12) \\
41,7 \%(50) \\
38,3 \%(46) \\
10,0 \%(12)\end{array}$ & $\begin{array}{l}7,5 \%(9) \\
25,0 \%(30) \\
47,5 \%(57) \\
20,0 \%(24)\end{array}$ & $0,014^{*}$ \\
\hline $\begin{array}{l}\text { Tipo de parto } \\
\text { Natural } \\
\text { Cesariana } \\
\text { Fórceps }\end{array}$ & $\begin{array}{l}31,1 \%(38) \\
28,7 \%(35) \\
40,2 \%(49)\end{array}$ & $\begin{array}{l}32,0 \%(39) \\
33,6 \%(41) \\
34,4 \%(42)\end{array}$ & $0,599^{*}$ \\
\hline
\end{tabular}

e ingestão de vísceras, legumes, frutas e cereais com um ano de idade. No grupo de filhos de adolescentes, sete crianças foram consideradas não amamentadas: um filho de mãe HIV positivo e seis outras que mamaram menos de vinte dias; enquanto que, entre os filhos de mães adultas, duas crianças não foram amamentadas devido à mastite grave materna e outras três mamaram menos que vinte dias. As mães que amamentaram menos que vinte dias estavam com problemas pessoais e familiares, que as desmotivaram a manter a amamentação. Detectou-se que os filhos de mães adolescentes ingerem significativamente menos carne e tendem a ingerir mais ovos que os filhos de mães adultas.

Usando o mesmo método de análise da amamentação exclusiva, foram analisadas: época do início da amamenta- ção predominante, amamentação completa e amamentação total no primeiro ano de vida, cujas médias e medianas com intervalos de confiança de $95 \%$, usando-se o método de análise de sobrevida de Kaplan-Meier, estão descritas na Tabela 4. A mediana da amamentação exclusiva foi de 90 dias para ambos os grupos, enquanto que a mediana da amamentação total foi de seis meses para as mães adolescentes, e de oito para as adultas.

As curvas comparativas de amamentação exclusiva, início da amamentação predominante, amamentação completa e total no primeiro ano de vida, entre os grupos, estão apresentadas nas Figuras 1, 2, 3 e 4, sem diferença estatisticamente significativas, aplicando-se o teste de Wilcoxon ou Breslow. 
Tabela 2 - Análise descritiva dos filhos de mães adolescentes versus filhos de mães adultas

\begin{tabular}{|c|c|c|c|}
\hline Características da criança & $\begin{array}{c}\text { Filhos de adolescentes } \\
\text { \% (n) }\end{array}$ & $\begin{array}{c}\text { Filhos de adultas } \\
\% \text { (n) }\end{array}$ & $\mathbf{p}$ \\
\hline Sexo & & & $0,655^{*}$ \\
\hline Masculino & $51,6 \%(63)$ & $48,8 \%(60)$ & \\
\hline Feminino & $48,4 \%(59)$ & $51,2 \%(63)$ & \\
\hline Adequação P / IG & & & $0,042 *$ \\
\hline $\mathrm{AIG}^{*}$ & $95,1 \%(116)$ & $87,8 \%(108)$ & \\
\hline $\mathrm{GIG}^{*}$ & $4,9 \%(6)$ & $12,2 \%(15)$ & \\
\hline APGAR do $5^{\circ}$ minuto $\geq 7$ & $98,4 \%(120)$ & $98,4 \%(121)$ & \\
\hline Hospitalização no $1^{\circ}$ ano de vida & $17,2 \%(21)$ & $14,6 \%(18)$ & $0,581 *$ \\
\hline Uso de creche no $1^{\circ}$ ano de vida & $7,4 \%(9)$ & $19,5 \%(24)$ & $\mathbf{0 , 0 0 5 *}$ \\
\hline Média de peso ao nascer (g) & $3.227 \pm 363,4$ & $3.291 \pm 429,2$ & $0,169^{\dagger}$ \\
\hline Comprimento ao nascer $(\mathrm{cm})$ & $49,2 \pm 1,7$ & $49,7 \pm 1,9$ & $\mathbf{0 , 0 2 7} 7^{\dagger}$ \\
\hline Perímetro craniano ao nascer $(\mathrm{cm})$ & $34,3 \pm 1,4$ & $34,4 \pm 1,5$ & $0,647^{\dagger}$ \\
\hline Capurro somático & $39,6 \pm 1,1$ & $39,8 \pm 1,11$ & \\
\hline Média de peso com 1 ano $(\mathrm{g})$ & $9.813 \pm 1.095$ & $10.138 \pm 1.186$ & $0,019^{\dagger}$ \\
\hline Média de comprimento com 1 ano $(\mathrm{cm})$ & $76,8 \pm 2,9$ & $77,0 \pm 2,67$ & $0,394^{\dagger}$ \\
\hline Média de perímetro cefálico com 1 ano $(\mathrm{cm})$ & $46,3 \pm 1,2$ & $46,5 \pm 1,3$ & $0,184^{\dagger}$ \\
\hline Média de perímetro braquial (cm) & $15,4 \pm 1,1$ & $15,8 \pm 1,2$ & $0,120^{\dagger}$ \\
\hline Média de prega tricipital (mm) & $8,6 \pm 2,1$ & $9,0 \pm 2,1$ & $0,130^{\dagger}$ \\
\hline Média de prega subescapular (mm) & $6,8 \pm 1,7$ & $6,9 \pm 1,7$ & $0,589^{\dagger}$ \\
\hline Média de IMC II & $16,6 \pm 1,4$ & $16,9 \pm 1,5$ & $0,041^{\dagger}$ \\
\hline
\end{tabular}

* Teste do qui-quadrado.

$\dagger$ ANCOVA corrigindo o efeito do sexo.

Ғ Adequação de peso para a idade gestacional: $\mathrm{AIG}=$ adequado para a idade gestacional, $\mathrm{GIG}=$ grande para a idade gestacional . ๆ $\mathrm{IMC}=$ índice de massa corporal.

\section{Discussão}

A amamentação não se mostrou diferente entre os grupos, reforçando Valenzuela ${ }^{20}$ e contrariando outros trabalhos que mostram que mães adolescentes têm maior adesão à amamentação que mães adultas ${ }^{21,22}$. Estudos recentes mostram que as melhores taxas de adesão à amamentação devem-se às intervenções de profissionais de saúde no pré-natal, no puerpério imediato e na puericultu$\mathrm{ra}^{23,24}$; e ainda mostram que as gestantes e mães adolescentes e as primíparas foram as que mais se beneficiaram da atividade educativa de promoção da amamentação ${ }^{23,24}$, reforçando a importância das atividades educativas, que podem ser desenvolvidas nos serviços de atenção primária à saúde. Também se observa influência positiva das avós, promovendo a amamentação ${ }^{11,25}$, enquanto que, estudo de Giugliani et al. ${ }^{26}$ sugere que elas interferem negativamente na duração do aleitamento.

A mediana da amamentação exclusiva para ambos os grupos foi de 90 dias, melhor que a encontrada em Montes Claros-MG, de apenas 27 dias $^{27}$. Lá, a mediana do aleitamento materno total foi 8,7 meses e, neste estudo, seis meses para as mães adolescentes e oito para as adultas.
Embora seja uma diferença não desprezível (dois meses), não houve diferença estatisticamente significante entre as curvas de aleitamento materno dos dois grupos (teste de Wilcoxon ou Breslow: $\mathrm{p}=0,269$ ). Pesquisadores mostram taxas de aleitamento exclusivo e predominante de $0,0 \%$ e $62,9 \%$ em Belém-PA ${ }^{11}$, em 1997; 10,3\% e $32,3 \%$ em Embu-SP ${ }^{12}$, em 1999; e de 37,8\% e 17,8\% em São Carlos$\mathrm{SP}^{13}$, em 2000; respectivamente. Essa diversidade de prevalências de aleitamento exclusivo e predominante mostra a influência dos hábitos culturais regionais e a necessidade de se promover programas de incentivo à amamentação adequados à cada região, uma vez que o Brasil é um país continental, rico em culturas diferentes, necessitando de abordagens regionalizadas para promoção da amamentação exclusiva. Não cabe um modelo único de promoção do aleitamento materno num país com tanta diversidade sociocultural.

O aleitamento continuado um ano foi de $35,3 \%$ e $28,5 \%$ para filhos de mães adolescentes e de adultas, respectivamente, enquanto que, em Belém-PA ${ }^{11}$ foi de $62,6 \%, 45,4 \%$ em Feira de Santana-BA ${ }^{28}, 27,6 \%$ em Embu-SP ${ }^{12}$, e de $41,0 \%$ em Montes Claros- $\mathrm{MG}^{27}$, mantendo-se a diversidade regional. 
Tabela 3 - Perfil de alimentação no primeiro ano de vida dos filhos de mães adolescentes e mães adultas

\begin{tabular}{|c|c|c|c|}
\hline Características da criança & $\begin{array}{c}\text { Filhos de adolescentes } \\
\%(\mathbf{n})\end{array}$ & $\begin{array}{c}\text { Filhos de adultas } \\
\%(\mathbf{n})\end{array}$ & $\mathbf{p}$ \\
\hline Amamentação & $115(94,3 \%)$ & $118(95,9 \%)$ & $0,544 *$ \\
\hline Aleitamento continuado - 1 ano & $43(35,3 \%)$ & $35(28,5 \%)$ & $0,254 *$ \\
\hline $\begin{array}{l}\text { Com } 1 \text { ano idade, leite materno } \\
\text { Leite materno + vaca } \\
\text { Leite de vaca }\end{array}$ & $\begin{array}{l}14(11,5 \%) \\
29(23,8 \%) \\
79(64,7 \%)\end{array}$ & $\begin{array}{l}11(9,0 \%) \\
24(19,5 \%) \\
90(71,5 \%)\end{array}$ & $0,519 *$ \\
\hline $\begin{array}{l}\text { Carnes diariamente } \\
\text { Ocasionalmente } \\
\text { Não ingere }\end{array}$ & $\begin{array}{l}17(13,9 \%) \\
104(85,3 \%) \\
1(0,8 \%)\end{array}$ & $\begin{array}{l}32(26,0 \%) \\
90(73,2 \%) \\
1(0,8 \%)\end{array}$ & $\mathbf{0 , 0 3 1} 1^{\dagger}$ \\
\hline $\begin{array}{l}\text { Vísceras ocasionalmente } \\
\text { Não ingere }\end{array}$ & $\begin{array}{l}81(66,4 \%) \\
41(33,6 \%)\end{array}$ & $\begin{array}{l}91(73,9 \%) \\
32(26,1 \%)\end{array}$ & $0,194 *$ \\
\hline $\begin{array}{l}\text { Ovos ocasionalmente } \\
\text { Não ingere }\end{array}$ & $\begin{array}{l}108(88,5 \%) \\
14(11,5 \%)\end{array}$ & $\begin{array}{l}99(80,5 \%) \\
24(19,5 \%)\end{array}$ & $0,082 *$ \\
\hline $\begin{array}{l}\text { Legumes diariamente } \\
\text { Ocasionalmente } \\
\text { Não ingere }\end{array}$ & $\begin{array}{l}82(67,2 \%) \\
40(32,8 \%) \\
\quad-\end{array}$ & $\begin{array}{l}86(69,9 \%) \\
35(28,5 \%) \\
2(1,6 \%)\end{array}$ & $0,349^{\dagger}$ \\
\hline $\begin{array}{l}\text { Frutas diariamente } \\
\text { Ocasionalmente } \\
\text { Não ingere }\end{array}$ & $\begin{array}{l}80(65,6 \%) \\
40(32,8 \%) \\
2(1,6 \%)\end{array}$ & $\begin{array}{l}90(73,2 \%) \\
29(23,6 \%) \\
4(3,2 \%)\end{array}$ & $0,216^{\dagger}$ \\
\hline $\begin{array}{l}\text { Cereais diariamente } \\
\text { Ocasionalmente } \\
\text { Não ingere }\end{array}$ & $\begin{array}{l}109(89,4 \%) \\
12(9,8 \%) \\
1(0,8 \%)\end{array}$ & $\begin{array}{c}110(89,4 \%) \\
13(10,6 \%) \\
-\end{array}$ & $1,000^{\dagger}$ \\
\hline
\end{tabular}

Comparando-se a escolaridade das mães adolescentes e das adultas, não se observou diferença, tendo-se o cuidado de adequar anos escolares com idade materna, evitando-se o viés de se nivelar adolescentes com poucos anos e adultas com escolaridade completa. Esta é uma variável importante, uma vez que se tem observado uma retomada do aleita- mento materno nos grupos de nível educacional mais elevados, após a fase de declínio decorrente da inserção da mulher no mercado de trabalho ${ }^{29}$.

Ao engravidar, as adolescentes apresentaram maior proporção de solteiras e/ou separadas, o que se alterou à época da entrevista, quando mudaram significativamente

Tabela 4 - Perfil de amamentação dos filhos de mães adolescentes e de adultas durante o primeiro ano de vida

\begin{tabular}{|c|c|c|c|c|c|}
\hline \multirow[t]{2}{*}{ Amamentaçãa } & \multicolumn{2}{|c|}{ Filhos de mães adolescentes } & \multicolumn{2}{|c|}{ Filhos de mães adultas } & \multirow[t]{2}{*}{$\mathbf{p}$} \\
\hline & Média * & Mediana * & Média * & Mediana* & \\
\hline Amamentação exclusiva & $\begin{array}{c}97 \\
(85,0-109,0)\end{array}$ & $\begin{array}{c}90 \\
(75,0-105,0)\end{array}$ & $\begin{array}{c}94 \\
(82,0-105,0)\end{array}$ & $\begin{array}{c}90 \\
(74,0-106,0)\end{array}$ & 0,632 \\
\hline $\begin{array}{l}\text { Época do início da } \\
\text { amamentação predominante }\end{array}$ & $\begin{array}{c}1,5 \\
(1,1-1,8)\end{array}$ & 1 & $\begin{array}{c}1,6 \\
(1,3-1,9)\end{array}$ & 1 & 0,076 \\
\hline Amamentação completa & $\begin{array}{c}103,4 \\
(93,4-113,4)\end{array}$ & $\begin{array}{c}90 \\
(71,6-108,4)\end{array}$ & $\begin{array}{c}111,6 \\
(101,7-121,5)\end{array}$ & $\begin{array}{c}120 \\
(111,6-128,4)\end{array}$ & 0,245 \\
\hline $\begin{array}{c}\text { Tempo total da amamentação } \\
\dagger\end{array}$ & $\begin{array}{c}202,7 \\
(176,5-228,9)\end{array}$ & $\begin{array}{c}180 \\
(123,2-236,8)\end{array}$ & $\begin{array}{c}228,8 \\
(203,2-254,4)\end{array}$ & $\begin{array}{c}240 \\
(192,2-287,8)\end{array}$ & 0,269 \\
\hline
\end{tabular}

\footnotetext{
* As médias e medianas estão expressas em dias, exceto a época do início da amamentação predominante, que está expressa em meses.

† Intervalos de confiança de 95\% das médias e medianas da amamentação, usando-se o método de análise de sobrevida de KaplanMeier.

‡ p valor das comparações usando teste de Wilcoxon ou Breslow.
} 


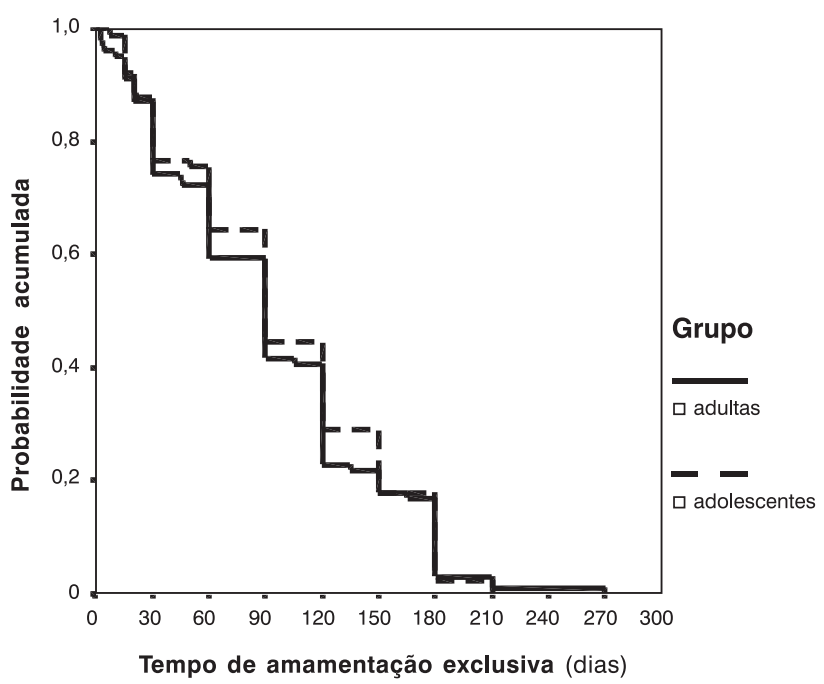

Figura 1 - Análise do tempo de amamentação exclusiva entre os grupos (teste de Wilcoxon ou Breslow: $p=0,632$ )

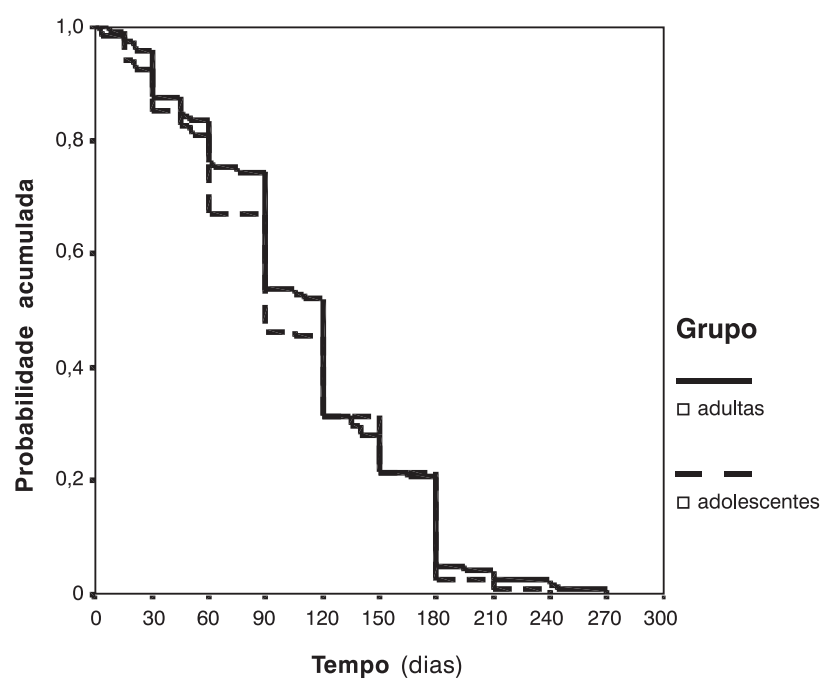

Figura 3 - Análise do tempo de amamentação completa (teste de Wilcoxon ou Breslow: $p=0,245$ )

de situação, amasiando-se ou casando-se, geralmente com o pai da criança ou homens mais velhos. A mudança de situação conjugal é importante para a estabilidade emocional e financeira dessas mães, caracterizando-se em fator positivo para o desenvolvimento de seus filhos.

O número de mães fumantes, em ambos grupos, foi pequeno e sem diferença estatisticamente significativa, dado diferente de estudo recente que mostra, no período de lactação, que o tabagismo decresce à proporção que o grau de escolaridade e nível de renda aumentam ${ }^{30}$; e outro aponta os malefícios do fumo, afetando a produção de leite

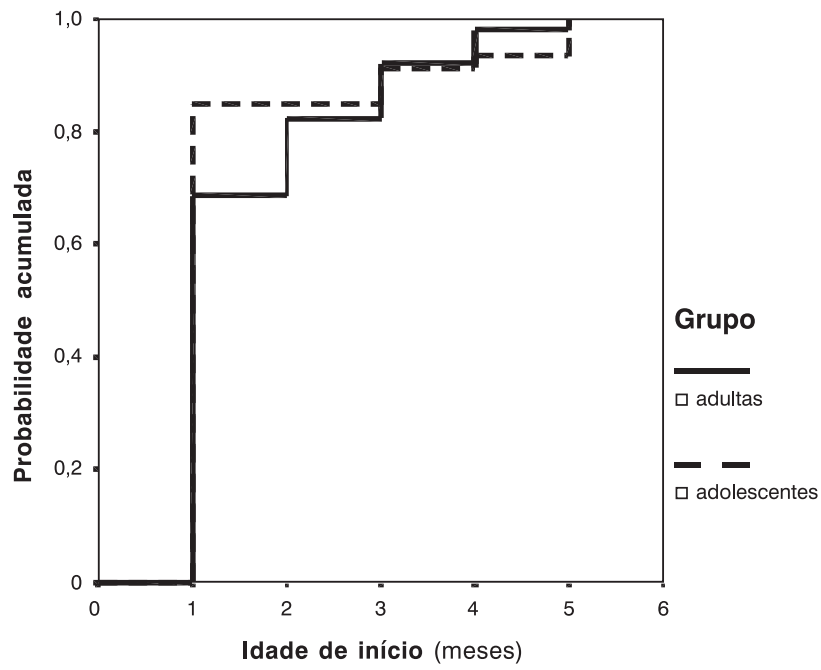

Figura 2 - Análise da idade de início da amamentação predominante (teste de Wilcoxon ou Breslow: $\mathrm{p}=0,077$ )

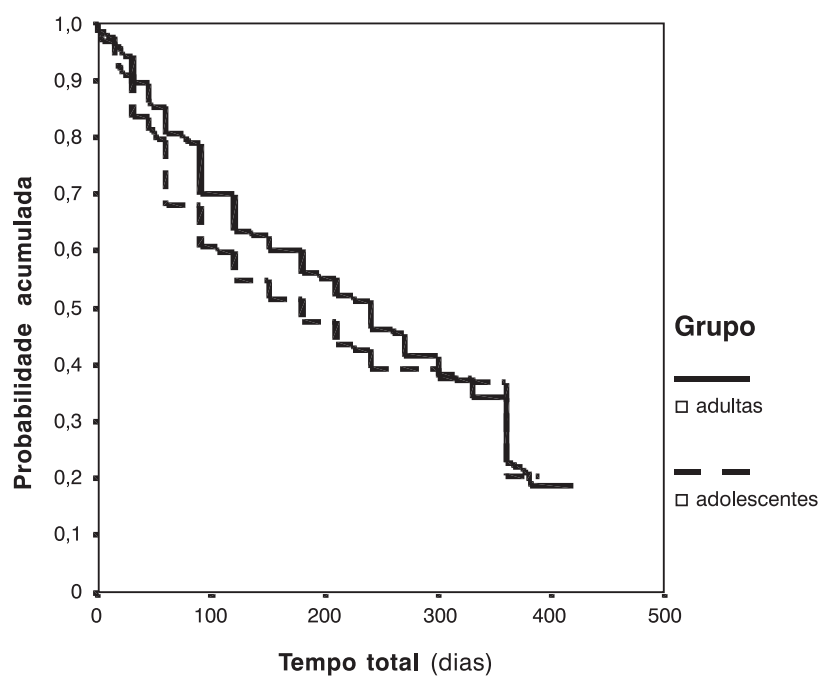

Figura 4 - Análise do tempo total da amamentação durante o primeiro ano de vida (teste de Wilcoxon ou Breslow: $\mathrm{p}=0,269)$

materno e o desenvolvimento infantil de crianças amamentadas, filhas de mães fumantes ${ }^{9}$.

Constatou-se que as mães adultas trabalham mais fora do lar, para ajudar no orçamento familiar, conseguindo maior renda per capita que as mães adolescentes, o que interfere na aquisição de alimentos, importantes para um adequado crescimento e desenvolvimento de seus filhos, também observado por Lima ${ }^{31}$ et al. Estudo mostra associação entre alto nível socioeconômico e educacional materno à escolha e maior duração do aleitamento materno ${ }^{25}$, mas, apesar das mães adolescentes desta amostra serem 
mais pobres que as mães adultas, não houve diferença com relação à escolaridade e amamentação.

A família é responsável pelo comportamento alimentar da criança através da aprendizagem social, influenciada por fatores psicossociais e culturais. Neste estudo, o fator socioeconômico provavelmente pesou nas práticas alimentares durante o primeiro ano de vida, pois os filhos de adolescentes, que têm menor renda, ingeriram significativamente menos carne que os filhos de adultas, sem diferença na ingestão dos outros alimentos complementares mais baratos.

Este estudo contribuiu para desmistificar a relação, dita desfavorável pela literatura, entre adolescência/amamentação/alimentação no primeiro ano de vida, uma vez que não se observou diferenças estatisticamente significativa entre os grupos, exceto menor consumo de carnes e maior ingestão de ovos pelos filhos de mães adolescentes, que apresentaram menor renda per capita e, conseqüentemente, menor poder aquisitivo para comprar carnes.

\section{Referências bibliográficas}

1. Guimarães EBM. Adolescência para pais e professores. $2^{\mathrm{a}}$ ed. Goiânia: Escaleno; 1992.

2. Uzcátegui O. El embarazo en la adolescente precoz. Rev Obstet Ginecol Venez 1997;57:29-35.

3. Victora CG, Smith PG, Vaughan JP, Nobre LC, Lombardi C, Teixeira AM, et al. Evidence for protection by breast-feeding against infant deaths from infectious diseases in Brazil. Lancet 1987;2(8554):319-22.

4. Gouvêa LC. Aleitamento materno. In: Nóbrega FJ, editor. Distúrbios da Nutrição. Rio de Janeiro: Revinter; 1998. p. 15-31.

5. Sociedade Civil Bem-Estar Familiar no Brasil. Pesquisa Nacional de Demografia e Saúde - 1996. Amamentação e Situação Nutricional das Mães e Crianças [site na Internet]. Disponível: www.amamentacao.org.br. Acessado: 13 de fevereiro de 2003.

6. Lindblad BS. What are the most effective means of promoting exclusive breastfeeding? Acta Paediatr 2000;89:1405-7.

7. Almroth S, Mohale M, Latham MC. Unnecessary water supplementation for babies: grandmothers blame clinics. Acta Paediatr 2000;89:1408-13.

8. World Health Organization/UNICEF. Innocenti Declaration on the protection, promotion and support of breastfeeding. Ecol Food Nutr 1991;26:271-3.

9. Giugliani ERJ. O aleitamento materno na prática clínica. J Pediatr (Rio J) 2000;76 Supl 2:238-52.

10. Giugliani ERJ, Victoria CG. Alimentação complementar. J Pediatr (Rio J.) 2000;76 Supl 2:253-62.

11. Moura EFA. Duração do período de aleitamento materno de crianças atendidas em ambulatório de pediatria. J Pediatr (Rio J) 1997;73:106-10.

12. Pedroso GC. Prevalência do aleitamento materno no município de Embu (São Paulo), no ano de 1996 [dissertação de mestrado]. São Paulo (SP): Escola Paulista de Medicina; 1999.

13. Montrone VG, Arantes CIS. Prevalência do aleitamento materno na cidade de São Carlos, São Paulo. J Pediatr (Rio J) 2000;76: 138-42.

14. Kramer MS, Kakuma R. Optimal duration of exclusive breastfeeding. Cochrane Database Syst Rev 2002;(1):CD003517.
15. Cohen RJ, Brown KH, Canahuati J, Rivera LL, Dewey KG. Effects of age of introduction of complementary food on infant breast milk intake, total energy intake, and growth: a randomized intervention study in Honduras. Lancet 1994;344:288-93.

16. Mehta KC, Specker BL, Bartholmey S, Giddens J, Ho ML. Trial on timing of introduction to solids and food type on infant growth. Pediatrics 1998;102:569-73.

17. Burrows R, Leiva L, Bravo R, Macho L, Zvaighaft A, Muzzo S. Crecimiento y desarrollo de hijos de adolescentes de clase mediabaja. II Parte: 30 meses de vida. Rev Chil Pediatr 1986;57:491-6.

18. Carruth BR, Nevling W, Skinner JD. Developmental and food profiles of infants born to adolescent and adult mothers. $\mathrm{J}$ Adolesc Health 1997;20:434-44.

19. Frisancho AR. Anthropometric standards for the assessment of growth and nutritional status. $4^{\mathrm{a}} \mathrm{ed}$. Ann Arbor: University of Michigan Press; 1993.

20. Valenzuela MS, Abarca BC, Aillach EB, Aliste VC, Alvarado CS, Bocic GA, et al. Primer año de vida en hijos de adolescentes. Boletín del Hospital San Juan de Dios 1987;34:81-5.

21. Burrows R, Carrasco D, Muzzo S. Crecimiento y desarrollo en hijos de adolescentes de clase media. Parte I. Rev Chil Pediatr 1985;56:199-204.

22. Romero MI, Yetzen G, Molina R, Alarcón G, González E, Arestizábal E, et al. Caracteristicas del primer año de vida de hijos de madres adolescentes: un estudio descriptivo de seguimiento. Cuadernos Medico Sociales 1984;25:124-9.

23. Susin LRO, Giugliani ERJ, Kummer SC, Maciel M, Benjamin $\mathrm{ACW}$, Machado DB, et al. Uma estratégia simples que aumenta os conhecimentos das mães em aleitamento materno e melhora as taxas de amamentação. J Pediatr (Rio J) 1998;74:368-76.

24. Bracco Neto H, Taddei JAAC. Mudança de conhecimento de gestantes em aleitamento materno através de atividade educacional. Revista Paulista de Pediatria 2000;18:7-14.

25. Primo CC, Caetano LC. A decisão de amamentar da nutriz: percepção de sua mãe. J Pediatr (Rio J) 1999;75:449-55.

26. Giugliani ER, Issler RM, Justo EB, Seffrin CF, Hartmann RM, Carvalho NM. Risk factors for early termination of breastfeeding in Brazil. Acta Paediatr 1992;81:484-7.

27. Caldeira AP, Goulart EMA. A situação do aleitamento materno em Montes Claros, Minas Gerais: estudo de uma amostra representativa. J Pediatr (Rio J) 2000;76:65-72.

28. Vieira GO, Glisser M, Araújo SPT, Sales NA. Indicadores do aleitamento materno na cidade de Feira de Santana, Bahia. J Pediatr (Rio J) 1998;74:11-6.

29. Bracco Neto H. Implantação e avaliação de atividades de incentivo ao aleitamento materno, dirigida a gestantes, em unidades básicas de saúde [dissertação de mestrado]. São Paulo (SP): Escola Paulista de Medicina; 1993.

30. Mello PRB, Pinto GR, Botelho C. Influência do tabagismo na fertilidade, gestação e lactação. J Pediatr (Rio J) 2001;77:257-64.

31. Lima M, Figueira F, Ebrahim GJ. Malnutrition among children of adolescent mothers in a squatter community of Recife, Brazil. J Trop Pediatr 1990;36:14-9.

Endereço para correspondência:

Dr. Antonio de Azevedo Barros Filho

Departamento de Pediatria da FCM - UNICAMP

Cx. Postal 6011 - CEP 13083-970 - Campinas, SP

Tels.: (19) 3788.7193 / 3788.7824 / 3788.7322

E-mail: abarros@fcm.unicamp.br 